\title{
Reversible cerebral vasoconstriction syndrome with cerebral infarction caused by acute high-level vapor exposure of ethylene oxide: a case report
}

\author{
Bin Lin ${ }^{1}$, Chao Wang ${ }^{1}$, Nan Lu' , Le Zhang ${ }^{2}$ and Biao Jiang ${ }^{\text {* }}$
}

\begin{abstract}
Background: With the increasing production and use of ethylene oxide (EO) worldwide, its explicit bio-toxicity has drawn more and more attention. At present, most studies focus on chronic EO exposure. Studies on acute EO exposure are rare, especially with imaging studies. To our knowledge, this work is the first documented case of reversible cerebral vasoconstriction syndrome (RCVS) with cerebral infarction caused by EO.
\end{abstract}

Case presentation: A 58-year-old woman who worked in a capsule production factory got an unprotected acute EO inhalation due to accidental exposure to sterilization gas. She suffered from nausea, vomiting, and severe paroxysmal headaches, but the first brain MRI scan of the patient showed no significant abnormality. Nine days after inhalation, she developed recurrent thunderclap headaches and gradual complete blindness. The follow-up brain MRI, 12 days after inhalation, demonstrated extensive cytotoxic edema. Fifteen days and 21 days after EO (ethylene oxide) inhalation, head MRA and CTA respectively showed diffuse vasoconstriction of cerebral arteries. Fifty-nine days after EO inhalation, head MRA assessed reversibility of the vasoconstriction. According to clinical features and imaging findings, RCVS with cerebral infarction can be diagnosed. The patient was sensitive to light and light reflection but still blind after symptomatic and rehabilitation therapy.

Conclusions: We report an acute EO exposure case in which the patient suffered from RCVS with cerebral infarction, which previous literature has not reported. This article aimed to raise awareness of encephalopathy after EO acute exposure.

Keywords: Ethylene oxide, Toxicology, Encephalopathy, Infarction, Headache

\section{Background}

Ethylene oxide (EO) is a colorless gas at room temperature with a pungent smell and colorless transparent liquid at about 12 degrees Celsius (53 degrees Fahrenheit). $\mathrm{EO}$ is an essential intermediate product in industrial

\footnotetext{
*Correspondence: jiangbiao@zju.edu.cn

${ }^{1}$ Department of Radiology, The Second Affiliated Hospital, Zhejiang

University School of Medicine, No.88 Jiefang Road, Hangzhou 310009,

Zhejiang, China

Full list of author information is available at the end of the article
}

production and a highly effective sterilizer at low temperatures. However, with the increasing production and use of EO worldwide, its explicit bio-toxicity has drawn more and more attention [1]. After inhalation or ingestion, the dissolved EO in the blood can often be metabolized and excreted within $24 \mathrm{~h}$ [2]. However, high levels of EO exposure can overwhelm the metabolic process and result in $\mathrm{EO}$ accumulation and its by-products resulting in toxic effects [3]. Symptoms in previously reported cases of EO acute exposure include dizziness, pulsatile headache, nausea, emesis, fatigue, paresthesia, drowsiness, ataxia, 
confusion, convulsions, mucous membrane irritation in eyes, nose, and respiratory tract, contact dermatitis and burns, some of which showing allergic reaction in type I or type IV with various clinical symptoms and even lead to death [4]. EO acute exposure is mainly caused by leakage due to operating procedures or unprotected operation [1]. At present, most studies focus on chronic EO exposure. There have not been many studies on EO acute exposure, especially those with diagnostic imaging that goes unreported.

\section{Case presentation}

A 58-year-old right-handed woman was a sterilization worker in a capsule production factory with nearly 10 years of service length. She suffered from nausea and vomiting after unprotected acute EO inhalation of leaked sterilization gas containing 75\% EO and $25 \%$ carbon dioxide in an air-locked room for an hour. The indoor concentrations of EO were uncertain. She had severe paroxysmal headaches, which were relieved when she came back home. The brain MRI of our hospital scan showed no abnormality 5 days after inhalation (Fig. 1, panel A-D). Nine days after inhalation, the patient developed recurrent thunderclap headache (striking suddenly like a clap of thunder and the severe headaches peaks within $60 \mathrm{~s}$ ) and partially lost bilateral eyesight in the whole field, which progressed to complete blindness and no light perception over the next $24 \mathrm{~h}$. A brain MRI scan performed again after admission, 12 days after inhalation, demonstrated extensive cytotoxic edema in the bilateral parietal lobe, occipital lobe, and left frontal lobe (Fig. 1, panel E-H). Fifteen days and 21 days after inhalation, Head MRA and CTA showed multiple areas of stenosis and partial occlusions involving the anterior, medial and posterior cerebral arteries bilaterally, respectively (Fig. 2). Laboratory tests: 12 days after inhalation, D-Dimer $880 \mu \mathrm{g} / \mathrm{L}(\mathrm{FEU}) \uparrow, \mathrm{GLB}-31.6 \mathrm{~g} / \mathrm{L} \uparrow, \mathrm{TG}-3.12 \mathrm{mmol} / \mathrm{L} \uparrow$. Subsequently, D-Dimer was continuously tested, this index through the process from rising to falling. 17, 32, 41, 51, 54 days after EO inhalation, D-Dimer value was 2920, 2500, 4960, 1630, and $760 \mu \mathrm{g} / \mathrm{L}(\mathrm{FEU}) \uparrow$, respectively. Pupillary light reflex was normal. The patient was alert and oriented to person, place, time, situation but

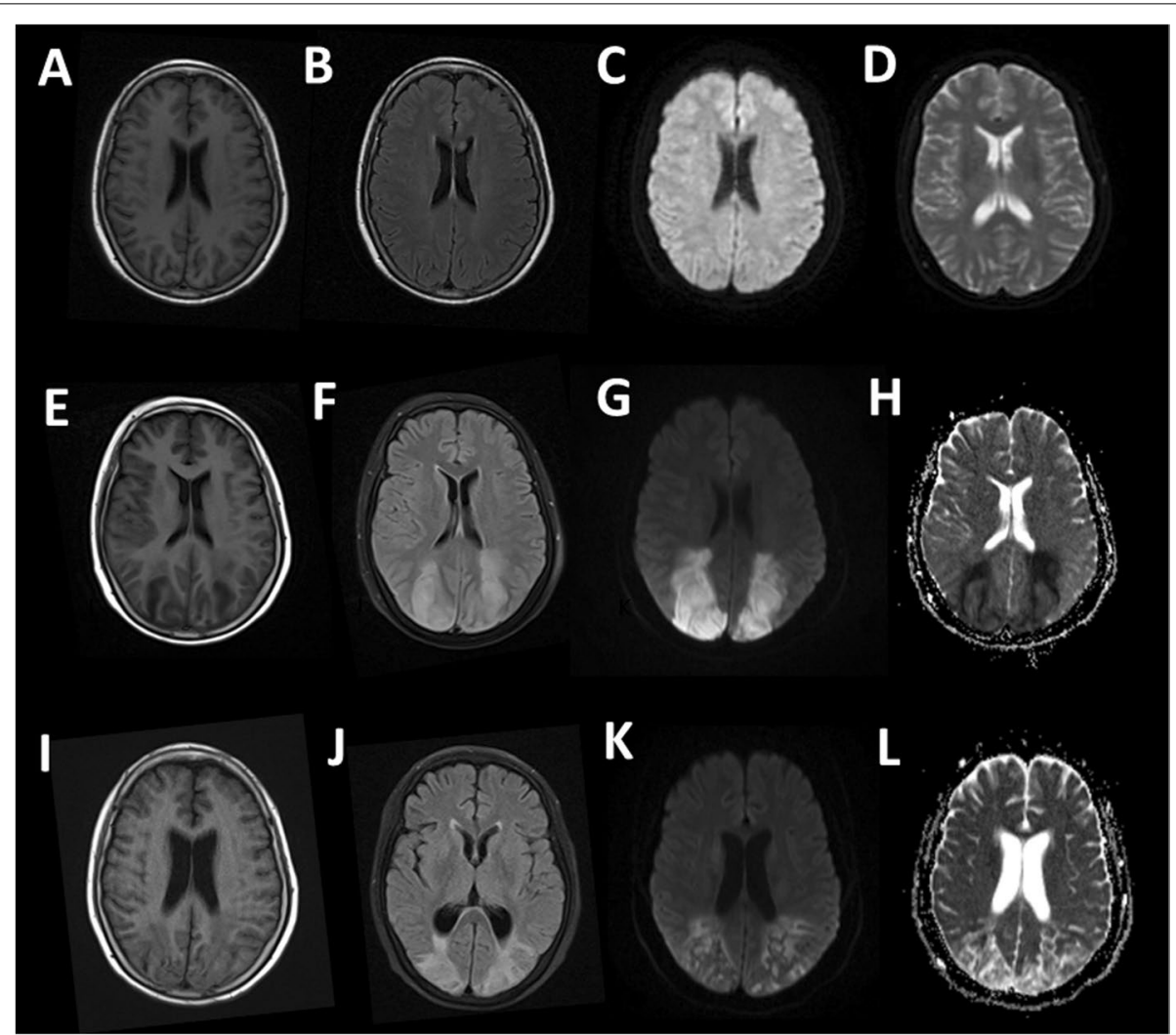

Fig. 1 Initial and follow-up axial T1WI, FLAIR, DWI, and ADC brain MRI. A-D: Axial T1WI, FLAIR, DWI, and ADC brain MRI performed 5 days after inhalation indicated that there was no noticeable abnormality. E-H: Axial T1WI, FLAIR, DWI, and ADC brain MRI performed 12 days after exposure demonstrates extensive cytotoxic edema in the parietal and occipital lobes bilaterally, left frontal lobe, centrum semiovale region. I-L: Axial T1WI, FLAIR, DWI, and ADC brain MRI performed 59 days after exposure demonstrates a notable improvement of the intracranial arterial stenosis 


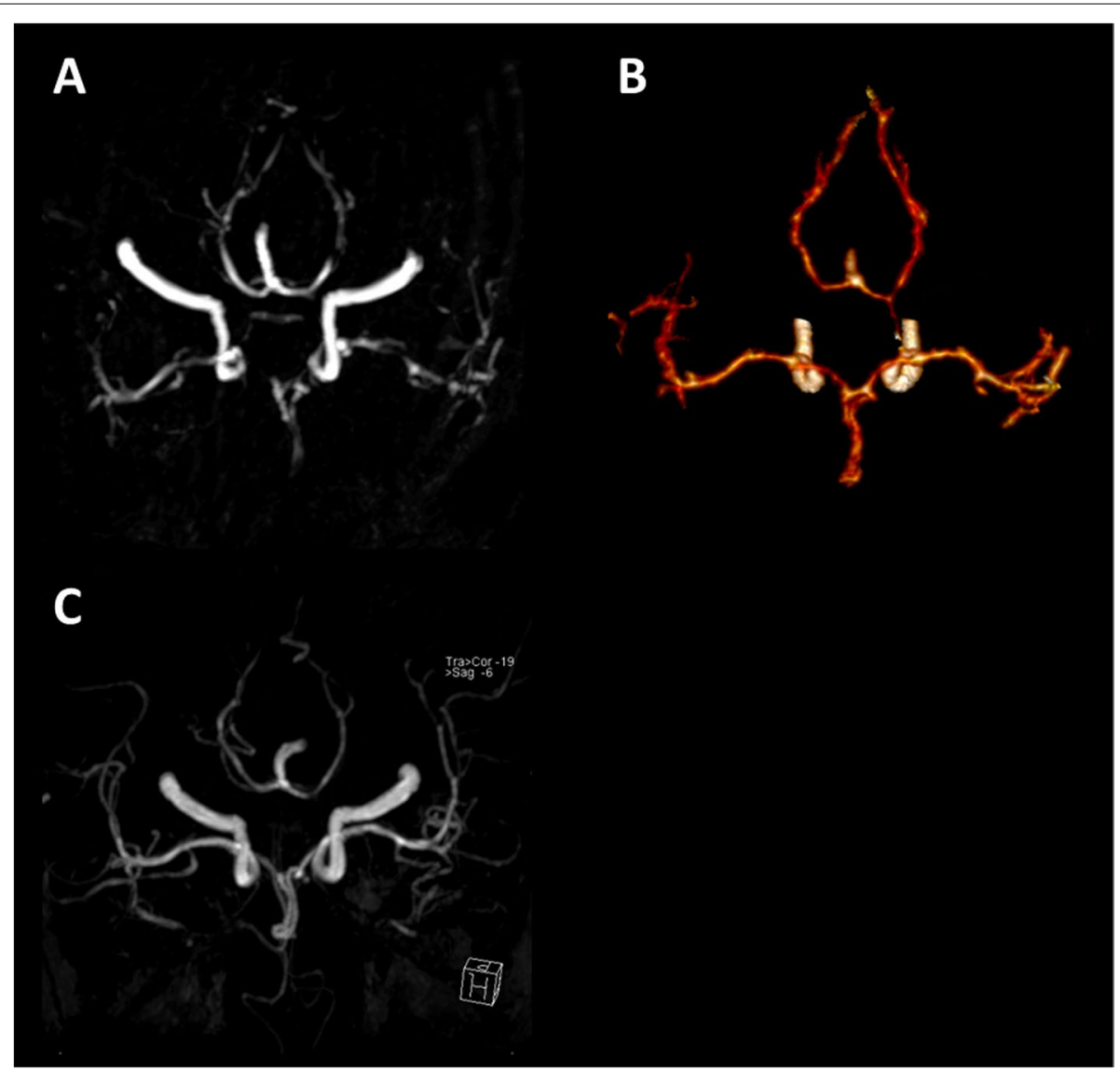

Fig. 2 Initial and follow-up CTA and MRA. The 15 days and 21 days after ethylene oxide inhalation, Head MRA (A) and CTA (B) showed multiple areas of stenosis and partial occlusions involving the anterior, medial and posterior cerebral arteries bilaterally, respectively. The 59 days after ethylene oxide inhalation, the follow-up head MRA (C) showed that the cerebral arterial stenosis was markedly improved but not complete recovery

slow to respond to questions. The patient had no history of high blood pressure, diabetes, hyperlipidemia, cerebrovascular disease. She did not drink alcohol or smoke and was without a family history of the inheritable disease. Currently, no consensus on the cut-off point for acute and chronic EO exposure. According to most definitions of intoxication, acute exposure is usually within 24 or $72 \mathrm{~h}$ of poisoning. Thus, the patient admitted with a presumptive diagnosis of "encephalopathy caused by EO acute exposure" because the patient developed clinical symptoms about $1 \mathrm{~h}$ after EO exposure.

During the hospitalization, the patient presented with left-sided hemiparesis and cortical blindness. After more than 2 months of symptomatic and rehabilitation therapy, the patient was sensitive to light but still blind after the therapy. Subsequently, the cerebral arterial stenosis markedly improved on a follow-up head MRA 59 days after inhalation (Fig. 2, panel C). Moreover, the DWI and ADC showed the lesions turned into softening lesions (Fig. 1, panel I-L).

\section{Discussion}

With the widespread use of ethylene oxide worldwide, its biotoxicity has attracted more and more attention. Ethylene oxide (EO) reacts with nearly all cellular components, including proteins, vitamins, cofactors, DNA, and RNA. Clinically, EO poisoning could cause acute neurological symptoms, including persistent nausea, vomiting, and recurrent thunderclap headache [5]. For the first time, we reported the case of reversible cerebral vasoconstriction syndrome (RCVS) with cerebral infarction caused by EO [6]. Specifically, the first-time brain MRI scan of the patient showed no significant abnormality. Next, 15 days and 21 days after EO inhalation, head MRA and CTA respectively showed diffuse vasoconstriction of cerebral arteries. Finally, 59 days after EO exposure, MRA assessed reversibility of the vasoconstriction was performed. According to clinical features and imaging findings, the diagnosis of reversible cerebral vasoconstriction syndrome (RCVS) is thus made. The patient did not receive any drug treatment with the nine-day intervals between EO exposure and recurrent thunderclap 
headache at onset and had no known exposure to other vasoconstrictors associated with RCVS. The patient's $\mathrm{RCVS}_{2}$ score was 7 , with a score of $>5$ having high specificity and sensitivity (99 and 90\%, respectively) for diagnosing RCVS (7). RCVS caused cerebral watershed infarction and posterior circulation infraction the brain MRI 12 days after inhalation showed. However, RCVS should be differentiated from another reversible neurological disease, the posterior reversible encephalopathy syndrome (PRES). These two diseases share many clinicoradiographic features, suggesting overlapping or similar pathophysiological mechanisms [5]. The PRES rarely causes stroke, although cerebral infarction, cytotoxic edema can occur in some cases [7]. The diagnosis of RCVS may be more reasonable. The imaging findings did, however, differ from other types of acute inhalation intoxication. Unlike toxic encephalopathy caused by other organic solvents and toxic gas inhalation, EO exposure does not involve the basal ganglia region, thalamus, dental nucleus, or cortical grey matter. It does not produce typical vasogenic cerebral edema or demyelination [8]. However, it may be similar to acute inhalation of vasoactive drugs such as cocaine which can cause RCVS [9].

Owing to its broad-spectrum antibacterial activity, EO is also used to sterilise hospital equipment and supplies that are not resistant to high temperature or high humidity, such as optical instruments, precision appliances, electronic components, and plastic capsulation. However, $\mathrm{EO}$ is a genotoxin and carcinogen with strong biotoxicity [10]. EO was recognized as one of the first carcinogens by the International Agency for Research on Cancer, an agency under the World Health Organization in 2017, and a hazardous air pollutant by the United States Environmental Protection Agency in November 2019.

\section{Conclusions}

The central nervous system is one of the primary targets of EO exposure, although the mechanism of neurotoxicity remains unclear. Finelli et al. postulated that EO might interfere with the metabolism of neuronal perikaryon or axonal transport, thus inhibiting the delivery of essential metabolites to nerve terminals [11]. RCVS from EO exposure has not been reported before. Some experimental animal studies are showing that EO exposure can cause vascular damage $[12,13]$. We speculate that the high concentration of EO inhalation caused vascular damage cerebral arteries of this patient and ultimately led to RCVS. Further research into the mechanism of injury on the central nervous system of acute EO exposure is necessary to determine potential vasoconstrictive effects on cerebral arteries and choose the optimal treatment for exposed patients.

\section{Abbreviations}

EO: Ethylene oxide; RCVS: Reversible cerebral vasoconstriction syndrome; $C T$ : Computed tomography; PRES: Posterior reversible encephalopathy syndrome; CTA: Computed tomography angiography; MRI: Magnetic resonance imaging; MRA: Magnetic resonance angiography.

\section{Acknowledgements}

The authors would like to thank the patient and his family members for generously permitting the use of the data.

\section{Authors' contributions \\ LB and ZL were responsible for medical data collection. LB and WC wrote the manuscript and analyzed image data. LN and JB drafted the work, analyzed image data, supervised the work, and substantively revised it. All authors read and approved the final manuscript.}

\section{Funding}

None.

\section{Availability of data and materials}

All data related to this case report are documented within this manuscript.

\section{Declarations}

Ethics approval and consent to participate

The ethics committee approved this study of The Second Affiliated Hospital, Zhejiang University School of Medicine.

\section{Consent for publication}

Written informed consent for publication of this Case Report was obtained from the patient. A copy of the written consent is available for review by the Editor of this journal.

\section{Competing interests}

The authors declare that they have no competing interests.

\section{Author details}

${ }^{1}$ Department of Radiology, The Second Affiliated Hospital, Zhejiang University School of Medicine, No.88 Jiefang Road, Hangzhou 310009, Zhejiang, China. ${ }^{2}$ Department of TCM Rehabilitation, The Second Affiliated Hospital, Zhejiang University School of Medicine, Hangzhou, China.

Received: 17 June 2021 Accepted: 4 October 2021

Published online: 09 October 2021

\section{References}

1. Marsh GM, Keeton KA, Riordan AS, Best EA, Benson SM. Ethylene oxide and risk of lympho-hematopoietic cancer and breast cancer: a systematic literature review and meta-analysis. Int Arch Occup Environ Health. 2019;92(7):919-39.

2. Mikoczy Z, Tinnerberg H, Björk J, Albin M. Cancer incidence and mortality in Swedish sterilant workers exposed to ethylene oxide: updated cohort study findings 1972-2006. Int J Environ Res Public Health. 2011;8(6):2009-19.

3. Ethrenberg L, Hiesche K, Osterman-Golkar S, Wennberg I. Evaluation of genetic risks of alkylating agents: tissue doses in the mouse from air contaminated with ethylene oxide. Mutat Res /Fundam Mol Mech Mutagen. 1974;24(2):83-103.

4. Gérin M, Tardif R. Urinary N-acetyl-S-2-hydroxyethyl-L-cysteine in rats as biological indicator of ethylene oxide exposure. Toxicol Sci. 1986;7(3):419-23.

5. Gross JA, Haas ML, Swift TR. Ethylene oxide neurotoxicity: report of four cases and review of the literature. Neurology. 1979;29(7):978.

6. Park RM. Associations between exposure to ethylene oxide, job termination, and cause-specific mortality risk. Am J Ind Med. 2020;63(7):577-88. 
7. Brashear A, Unverzagt F, Farber M, Bonnin J, Garcia J, Grober E. Ethylene oxide neurotoxicity: a cluster of 12 nurses with peripheral and central nervous system toxicity. Neurology. 1996;46(4):992-8.

8. Rocha EA, Topcuoglu MA, Silva GS, Singhal AB. RCVS2 score and diagnostic approach for reversible cerebral vasoconstriction syndrome. Neurology. 2019;92(7):e639-e47.

9. Ducros A. Reversible cerebral vasoconstriction syndrome. Lancet Neurol. 2012;11(10):906-17.

10. McKinney AM, Short J, Truwit CL, McKinney ZJ, Kozak OS, SantaCruz $\mathrm{KS}$, et al. Posterior reversible encephalopathy syndrome: incidence of atypical regions of involvement and imaging findings. Am J Roentgenol. 2007;189(4):904-12

11. Finelli PF, Morgan TF, Yaar I, Granger CV. Ethylene oxide-induced polyneuropathy: a clinical and Electrophysiologic study. Arch Neurol. 1983;40(7):419-21.
12. Snellings WM, Nachreiner DJ, Pottenger LH. Ethylene oxide: acute four-hour and one-hour inhalation toxicity testing in rats. J Toxicol. 2011:2011:910180.

13. Jin $X$, Wang T, Liao Y, Guo J, Wang G, Zhao F, et al. Neuroinflammatory reactions in the brain of 1, 2-DCE-intoxicated mice during brain edema. Cells. 2019;8(9):987.

\section{Publisher's Note}

Springer Nature remains neutral with regard to jurisdictional claims in published maps and institutional affiliations.
Ready to submit your research? Choose BMC and benefit from:

- fast, convenient online submission

- thorough peer review by experienced researchers in your field

- rapid publication on acceptance

- support for research data, including large and complex data types

- gold Open Access which fosters wider collaboration and increased citations

- maximum visibility for your research: over $100 \mathrm{M}$ website views per year

At BMC, research is always in progress.

Learn more biomedcentral.com/submissions 